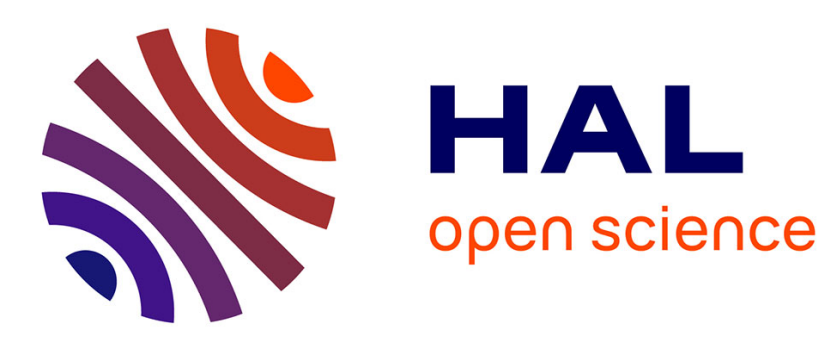

\title{
Large population asymptotics for interacting diffusions in a quenched random environment \\ Eric Luçon
}

\section{To cite this version:}

Eric Luçon. Large population asymptotics for interacting diffusions in a quenched random environment. Particle Systems and PDEs II, Dec 2013, Braga, Portugal. pp.231-251, 10.1007/978-3-31916637-7. hal-01022199

\section{HAL Id: hal-01022199 https://hal.science/hal-01022199}

Submitted on $10 \mathrm{Jul} 2014$

HAL is a multi-disciplinary open access archive for the deposit and dissemination of scientific research documents, whether they are published or not. The documents may come from teaching and research institutions in France or abroad, or from public or private research centers.
L'archive ouverte pluridisciplinaire HAL, est destinée au dépôt et à la diffusion de documents scientifiques de niveau recherche, publiés ou non, émanant des établissements d'enseignement et de recherche français ou étrangers, des laboratoires publics ou privés. 


\title{
Large population asymptotics for interacting diffusions in a quenched random environment
}

Eric Luçon

\begin{abstract}
We review some recent results on large population behavior of interacting diffusions in a random environment. Emphasis is put on the quenched influence of the environment on the macroscopic behavior of the system (law of large numbers and fluctuations). We address the notion of (non-)self-averaging phenomenon for this class of models. A guiding thread in this survey is the Kuramoto synchronization model which has met in recent years a growing interest in the literature.
\end{abstract}

Key words: Interacting diffusion, random environment, self-averaging, fluctuations, McKean-Vlasov equation, FitzHugh-Nagumo model, Kuramoto model, stochastic partial differential equation.

\section{Introduction}

\subsection{Diffusions in mean-field interaction}

In this paper, we review some recent results ([39, 40, 41]) concerning large population asymptotics of interacting diffusions in a random environment. This class of models generalizes systems of particles in a mean-field interaction that have been intensively studied since McKean [44]. A general instance of such mean-field models may be given as follows: for any fixed $T>0$, for any $N \geq 1$ and $m \geq 1$, consider the system of $N$ coupled stochastic differential equations in $\mathbf{R}^{m}$

$$
\mathrm{d} \theta_{i, t}=c\left(\theta_{i, t}\right) \mathrm{d} t+b\left(\theta_{i, t}, v_{N, t}\right) \mathrm{d} t+\sigma\left(\theta_{i, t}, v_{N, t}\right) \cdot \mathrm{d} B_{i, t}, t \in[0, T], i=1 \ldots, N,
$$

where $v_{N}$ is the empirical measure of the particles $\left(\theta_{1}, \ldots, \theta_{N}\right)$ :

\section{Eric Luçon}

Laboratoire MAP 5 (UMR CNRS 8145), Université Paris Descartes, Sorbonne Paris Cité, 45 rue des Saints-Pères, 75270 Paris cedex 06, France e-mail: eric.lucon@parisdescartes.fr 


$$
v_{N, t}:=\frac{1}{N} \sum_{i=1}^{N} \delta_{\theta_{i, t}}, \quad i=1, \ldots, N, t \in[0, T]
$$

For fixed $N$ and $t, v_{N, t}$ is an element of $\mathscr{M}_{1}\left(\mathbf{R}^{m}\right)$, the set of probability measures on $\mathbf{R}^{m}$. In the following, unless specified otherwise, $\mathscr{M}_{1}$ is endowed with its weak topology. In (1), $\left(B_{i}\right)_{i=1, \ldots, N}$ is a collection of independent Brownian motions in $\mathbf{R}^{p}$ $(p \geq 1),(\theta, v) \mapsto b(\theta, v) \in \mathbf{R}^{m}$ and $(\theta, v) \mapsto \sigma(\theta, v) \in \mathbf{R}^{m \times p}$ are (possibly not) regular functions of $(\theta, v)$, where $v$ belongs to the set of probability measures on $\mathbf{R}^{m}$. To fix ideas, the reader may think of the particular case where $b$ or $\sigma$ are of the form $(\theta, v) \mapsto \int \Gamma(\theta, \tilde{\theta}) v(\mathrm{~d} \tilde{\theta})$ where $\Gamma$ is a regular function. In such a case, the mean-field term in (1) reduces to $\frac{1}{N} \sum_{j=1}^{N} \Gamma\left(\theta_{i}, \theta_{j}\right)$.

Mean-field models like (1) have been quite popular in recent years in both physical and mathematical literature, as they provide the most natural way to represent the time evolution of a population of particles in all-to-all interaction. In (1), we have distinguished the local contribution $c\left(\theta_{i, t}\right) \mathrm{d} t$ to the dynamics (that would govern the dynamics if $\theta_{i}$ had not been connected to the rest of the population) from the mean-field contribution of all other particles $b\left(\theta_{i, t}, v_{N, t}\right) \mathrm{d} t$ modeling the interaction with the rest of the population.

A reason for the interest in such systems is that they also provide natural particle approximations for various partial differential equations appearing in physics (e.g. granular media equation [12, 42], porous media equation [11], Vicsek model for alignment of self-propelled particles [20]), biology (neuronal models [22, 4, 38], Keller-Segel model for chemotaxis [30]) or finance (rank-based models [34, 36]).

The problematic concerns the large population behavior of (1): under mild assumptions on the coefficients $c, b$ and $\sigma$, one can show that the empirical measure (2) converges weakly as $N \rightarrow \infty$ to a measure-valued solution $t \mapsto v_{t}$ of a nonlinear Fokker-Planck equation (or McKean-Vlasov equation [27, 44]). In that extent, a crucial feature of such mean-field systems is exchangeability: if, at $t=0$ the law of the vector $\left(\theta_{1,0}, \ldots, \theta_{N, 0}\right)$ is invariant under permutation, the same property holds for (1) at any positive time $t$. It is well understood ([53]) that, under this assumption, the above convergence is equivalent to the notion of propagation of chaos: for any fixed $k \geq 1$, the first $k$ particles $\left(\theta_{1}, \ldots, \theta_{k}\right)$ converges in law as $N \rightarrow \infty$ to $k$ independent copies of a nonlinear process, whose law is given by the solution to the McKean-Vlasov equation. More generally, a vast literature addresses the questions of fluctuations and large deviation of the empirical measure around its limit (see [26, 19, 27, 46, 24, 32] and references therein) as well as long-time behavior of such processes (uniform propagation of chaos and concentration inequalities $[13,12])$. 


\subsection{Inhomogeneous interacting diffusions}

\subsubsection{General framework}

The class of models we address in this paper is a generalization of (1) in the presence of a random environment. Namely, the system we consider is the following

$$
\mathrm{d} \theta_{i, t}=c\left(\theta_{i, t}, \omega_{i}\right) \mathrm{d} t+\frac{1}{N} \sum_{j=1}^{N} \Gamma\left(\theta_{i, t}, \omega_{i}, \theta_{j, t}, \omega_{i}\right) \mathrm{d} t+\mathrm{d} B_{i, t}, i=1, \ldots, N, t \in[0, T],
$$

endowed with an initial condition $\left(\theta_{i, 0}\right)_{i=1, \ldots, N}$ i.i.d. with law $\zeta$ on $\mathbf{R}^{m}$.

The difference between ( 3$)$ and (1) is that the particles $\left(\theta_{1}, \ldots, \theta_{N}\right)$ now live in a random environment, that is both the local dynamics $c(\cdot)$ and the mean-field term $\Gamma(\cdot)$ are perturbed by a given sequence $\left(\omega_{i}\right)_{i=1, \ldots, N} \in\left(\mathbf{R}^{n}\right)^{N}(n \geq 1)$ of i.i.d. random variables, independent of the thermal noise $\left(B_{1}, \ldots, B_{N}\right)$. This sequence models a local inhomogeneity in the system: particles are similar but not necessarily identical. The common law of the random variables $\left(\omega_{i}\right)_{i \geq 1}$ is denoted as $\mu \in \mathscr{M}_{1}\left(\mathbf{R}^{n}\right)$.

Remark 1. Note that we do not address the whole generality of the coefficients $b$ and $\sigma$ as described in (1). In particular, we restrict ourselves for simplicity to the case $p=m$ and $\sigma=I$. But the results exposed here should remain valid for general $\sigma$, provided further regularity and non-degeneracy assumptions are made.

From a point of view from statistical physics, the additional randomness $\left(\omega_{i}\right)_{i \geq 1}$ in (3) will be considered as a disorder. In this framework, there exist two ways to consider (3): one can either study the averaged (or annealed) model (where one looks at $\left(\theta_{1}, \ldots, \theta_{N}\right)$ under the joint law of the noise and the disorder) or one can fix once and for all a typical realization of the disorder and consider (3) under the law of the noise only (quenched model). In this paper, we focus on the quenched model, which is more realistic from a modeling point of view. Under the same hypotheses, the asymptotic results proven here remain valid in the (technically easier) averaged framework.

The difficulty of working in a quenched environment $\left(\omega_{i}\right)_{i>1}$ lies in the fact that the particles $\left(\theta_{1}, \ldots, \theta_{N}\right)$ are no longer exchangeable. In particular, the question of propagation of chaos concerning (3) is a priori not clear. More precisely, the question we want to address is the influence of a fixed realization of the disorder on the behavior of the empirical measure of the system as $N \rightarrow \infty$

$$
v_{N, t}=v_{N, t}^{(\omega)}:=\frac{1}{N} \sum_{i=1}^{N} \delta_{\left(\theta_{i, t}, \omega_{i}\right)}, \quad t \in[0, T], N \geq 1
$$

As in (4), we will specify when required the dependence of the empirical measure in the specific choice of the disorder $(\omega):=\left(\omega_{i}\right)_{i \geq 1}$. 


\subsubsection{Synchronization models and neural networks}

A first motivation for systems such as (3) comes from synchronization models in physics. The Kuramoto model [1], which will be the guiding thread of this review, is a particular case of ( 3 ) where the particles $\theta_{i}$ reduce to one-dimensional oscillators (or rotators) on the circle $\mathbf{S}:=\mathbf{R} / 2 \pi$, within a mean-field sine interaction, perturbed by random frequencies $\omega_{i} \in \mathbf{R}$ :

$$
\mathrm{d} \theta_{i, t}=\omega_{i} \mathrm{~d} t+\frac{K}{N} \sum_{j=1}^{N} \sin \left(\theta_{j, t}-\theta_{i, t}\right) \mathrm{d} t+\mathrm{d} B_{i, t}, \quad i=1, \ldots, N, t \in[0, T] .
$$

In (5), $K>0$ is the coupling strength between rotators, and the disorder $\omega_{i}$ is the local random frequency of rotator $\theta_{i}$, that may differ from one rotator to another. As $N \rightarrow \infty$, the system is described by the following nonlinear Fokker-Planck equation (whose solution $(t, \theta, \omega) \mapsto q_{t}(\theta, \omega)$ is the density of rotators at time $t$ at position $\theta$ with frequency $\omega)$ :

$$
\partial_{t} q_{t}=\frac{1}{2} \partial_{\theta}^{2} q_{t}(\theta, \omega)-\partial_{\theta}\left(q_{t}(\theta, \omega)\left(\omega+K \int_{\mathbf{S} \times \mathbf{R}} \sin (\tilde{\theta}-\theta) q_{t}(\tilde{\theta}, \tilde{\omega}) \mathrm{d} \tilde{\theta} \mu(\mathrm{d} \tilde{\omega})\right)\right) .
$$

The Kuramoto model was first introduced in [37] in order to study collective behavior of synchronizing individuals (neurons, social insects, cardiac cells) and has been since the subject of a vast literature, mostly in physics (see [37, 51, 1] and references therein) and more recently in mathematics.

The intuition for the dynamics of the Kuramoto model is simple: in a large population, each rotator $\theta_{i}$ obeys to the influence of its local frequency $\omega_{i}$ which tends to desynchronize the rotators, in contradiction with the mean-field coupling that tends to make the particles rotate together. A striking result, first observed by Kuramoto and Sakaguchi ([50]), is that (6) exhibits a phase transition: if $K$ is smaller than a critical value $K_{c}$, the uniform distribution $q \equiv \frac{1}{2 \pi}$ is the only stationary solution of (6) (there is no synchronization), whereas it coexists with nontrivial synchronized profiles for $K>K_{c}$. Recent results address the question of the long-time stability of such synchronized solutions, in the case without disorder $([8,9,29])$ and also with small disorder ([28]).

A second motivation for this work comes from the modeling of the spiking activity of neurons in a noisy environment. The FitzHugh-Nagumo model, which is a 2-dimensional simplification of the Hodgkin-Huxley model (see [4, 23] for further neurophysiological insights on the subject) is given as follows: $\theta_{i}:=\left(V_{i}, w_{i}\right)$ and

$$
\left\{\begin{array}{l}
\mathrm{d} V_{i}(t)=\left(V_{i}(t)-\frac{V_{i}(t)^{3}}{3}-w_{i}(t)+I\right) \mathrm{d} t, \\
\mathrm{~d} w_{i}(t)=\left(a_{i}\left(b_{i} V_{i}(t)-w_{i}(t)\right)\right) \mathrm{d} t,
\end{array} \quad i=1, \ldots, N, t \in[0, T],\right.
$$

where $a_{i}$ and $b_{i}$ are random coefficients. The variable $V_{i}(t)$ denotes the voltage activity of the neuron, $w_{i}(t)$ is a recovery variable and $I$ is the exterior input current. 
In (7), the disorder $\omega_{i}=\left(a_{i}, b_{i}\right) \in \mathbf{R}^{2}$ plays the role of a random discrimination between inhibited and excited neurons: it is well known ([23]) that, depending on the values of the parameters $\left(a_{i}, b_{i}\right)$, the dynamics (7) exhibits either periodic behavior around a limit cycle (spiking activity) or convergence to a fixed point (inhibition of the neuron). If one incorporates this dynamics into (3), the mean-field term $\Gamma(\cdot)$ models connections between neurons through electrical synapses. We refer to [4] for precise details and the exposition of more elaborate mean-field models applied to neuronal activity. Note that one difficulty of the dynamics given by (7) is that it is unbounded and not uniformly Lipschitz-continuous.

\subsubsection{Existing literature on disordered interacting diffusions}

The subject of diffusions in random environment has already been addressed in the literature. In particular, one should mention the seminal paper of Dai Pra and den Hollander [18] where an averaged large deviations principle for models similar to (3) is proved, with applications to the Kuramoto model and spin-flip systems. Other interesting applications of (3) may be found in the context of statistical physics (random Curie-Weiss model [16], model of social interactions [17]).

Instead of putting the disorder on the particles, it would also make sense to put the disorder on the connections between particles. There is currently a growing interest in mean-field models with random connectivities (in particular with applications to neuronal models, see $[25,55,14,54]$ and references therein), that is, models of the type

$$
\mathrm{d} \theta_{i, t}=\sum_{j=1}^{N} J_{i, j} \Gamma\left(\theta_{i, t}, \theta_{j, t}\right) \mathrm{d} t+\mathrm{d} B_{i, t}, \quad i=1, \ldots, N, t \in[0, T],
$$

where $\left(J_{i, j}\right)_{i, j=1, \ldots, N}$ is a (possibly symmetric) collection of random variables. Large population asymptotics of such models have been first studied through large deviations techniques by Guionnet and Ben Arous [3, 6] in the context of spin-glass systems. The long time analysis of the associated nonlinear process appears to be significantly more difficult than for the models considered here.

Other mean-field models of neurons with integrate-and-fire dynamics have recently been studied $[15,21,22]$. For such models, existence of a solution for all time to the nonlinear Fokker Planck turns out to be problematic.

\subsection{Organization of the paper}

We summarize in Section 2 the main results of the paper, that is a quenched law of large numbers and a quenched central limit theorem for the empirical measure (4). In Section 3, the main lines of proof for the two results are indicated. 


\section{Main results}

In the remaining of the paper, for any $p \geq 1$, the euclidean norm and scalar product in $\mathbf{R}^{p}$ are respectively denoted as $|u|$ and $u \cdot v\left(u, v \in \mathbf{R}^{p}\right)$. $\mathbf{P}$ is the law of the sequence of Brownian motions and $\mathbb{P}$ is the law of the sequence of the disorder. The corresponding expectations are denoted as $\mathbf{E}$ and $\mathbb{E}$ respectively.

\subsection{Quenched law of large numbers}

The first result concerns a quenched law of large numbers for the empirical measure $v_{N}$ defined in (4).

\subsubsection{Assumptions}

We assume that the coefficients and initial condition in (3) satisfy:

1. The function $(\theta, \omega) \mapsto c(\theta, \omega)$ is locally Lipschitz-continuous in $\theta$ (for fixed $\omega$ ) and satisfy a one-sided Lipschitz condition:

$$
\forall(\theta, \omega),(\bar{\theta}, \bar{\omega}),(\theta-\bar{\theta}) \cdot(c(\theta, \omega)-c(\bar{\theta}, \bar{\omega})) \leq L\left(|\theta-\bar{\theta}|^{2}+|\omega-\bar{\omega}|^{2}\right),
$$

for some constant $L$. The function $c$ also satisfies a polynomial bound:

$$
\forall(\theta, \omega),|c(\theta, \omega)| \leq C\left(1+|\theta|^{\kappa}+|\omega|^{l}\right),
$$

for some constant $C>0$ and where $\kappa \geq 2$ and $\imath \geq 1$.

2. The interaction term $\Gamma$ is bounded and globally Lipschitz on $\left(\mathbf{R}^{m} \times \mathbf{R}^{n}\right)^{2}$.

3. For fixed $\tilde{\theta}, \omega, \tilde{\omega}$, the functions $\theta \mapsto c(\theta, \omega)$ and $\theta \mapsto \Gamma(\theta, \omega, \tilde{\theta}, \tilde{\omega})$ are twice differentiable with continuous derivatives.

4. The initial distribution $\zeta$ of the particles in (3) and the law $\mu$ of the disorder satisfy the following moment conditions:

$$
\int_{\mathbf{R}^{m}}|\theta|^{\kappa} \zeta(\mathrm{d} \theta)<\infty \text { and } \int_{\mathbf{R}^{n}}|\omega|^{l} \mu(\mathrm{d} \omega)<\infty
$$

where the constants $\kappa$ and $l$ are given in (10).

Remark 2. These assumptions differ from the hypotheses of the original proof in [39]. Indeed, in [39], we were mostly concerned with particles living in a compact space (as in the Kuramoto model (5)), in which case assumptions on $c, \Gamma$ and $\zeta$ (especially the differentiability of $c$ and $\Gamma$ ) can be simplified. We refer to [39], Section 2.3.1 for further details. Note that the present assumptions specifically include the case of polynomial coefficients (as in the FitzHugh-Nagumo case (7), set $\kappa=3$ and $\imath=1$ ) which is not covered by [39]. 


\subsubsection{Law of large numbers and McKean-Vlasov equation}

Theorem 1 ([39], [41]). Under the hypotheses of Section 2.1.1,

1. there is a unique process $t \mapsto v_{t}$ in $\mathscr{C}\left([0, T], \mathscr{M}_{1}\left(\mathbf{R}^{m} \times \mathbf{R}^{n}\right)\right)$ such that $v_{0}=$ $\zeta \times \mu$ and $\sup _{t \leq T} \int\left(|\theta|^{\kappa} \vee|\omega|^{l}\right) v_{t}(\mathrm{~d} \theta, \mathrm{d} \omega)<+\infty$, satisfying the weak McKeanVlasov equation

$$
\left\langle v_{t}, f\right\rangle=\left\langle v_{0}, f\right\rangle+\int_{0}^{t}\left\langle v_{s}, \frac{1}{2} \Delta_{\theta} f+\nabla_{\theta} f \cdot\left(\left[\Gamma, v_{s}\right]+c\right)\right\rangle \mathrm{d} s, t \in[0, T],
$$

where $\langle v, f\rangle:=\int f(\theta, \omega) v(\mathrm{~d} \theta, \mathrm{d} \omega)$ and

$$
[\Gamma, m](\theta, \omega):=\int \Gamma(\theta, \omega, \tilde{\theta}, \tilde{\omega}) m(\mathrm{~d} \tilde{\theta}, \mathrm{d} \tilde{\omega}) .
$$

2. for almost-every sequence $\left(\omega_{i}\right)_{i \geq 1}$, the sequence $\left(v_{N}^{(\omega)}\right)_{N \geq 1}$ converges as $N \rightarrow \infty$ in $\mathscr{C}\left([0, T], \mathscr{M}_{1}\left(\mathbf{R}^{m} \times \mathbf{R}^{n}\right)\right)$ to the unique solution $v$ of $(12)$.

Remark 3. Note that if we take a test function $f$ in (12) that does not depend on $\theta$, we obtain that $\left\langle v_{0}, f\right\rangle=\left\langle v_{t}, f\right\rangle, \forall t \in[0, T]$. In particular, the marginal distribution on $\omega$ of $v_{t}$ is independent of $t$ and equal to $\mu$.

Since the noise in (3) is non-degenerate, using the regularizing properties of the heat kernel, one can prove that for any measure-valued initial condition in (12), the solution of (12) has a regular density $v_{t}(\mathrm{~d} \theta, \mathrm{d} \omega)=q_{t}(\theta, \omega) \mathrm{d} \theta \mu(\mathrm{d} \omega)$ for all $t>0$. Integrating by parts in (12), we find that $q_{t}$ is a strong solution to

$$
\begin{aligned}
\partial_{t} q_{t} & =\frac{1}{2} \Delta_{\theta} q_{t}(\theta, \omega)-\operatorname{div}_{\theta}\left(q_{t}(\theta, \omega) c(\theta, \omega)\right) \\
& -\operatorname{div}_{\theta}\left(q_{t}(\theta, \omega) \int \Gamma(\theta, \omega, \tilde{\theta}, \tilde{\omega}) q_{t}(\tilde{\theta}, \tilde{\omega}) \mathrm{d} \tilde{\theta} \mu(\mathrm{d} \tilde{\omega})\right), t>0 .
\end{aligned}
$$

For a proof of this fact and further details, we refer to [28, Prop. A.1].

A consequence of Theorem 1 is that, at the level of the law of large numbers, the system only depends on the law $\mu$ of the disorder, but not on a typical realization of the $\left(\omega_{i}\right)_{i \geq 1}$ : there is a self-averaging phenomenon. The notion of self-averaging (or its absence) is crucial in many disordered models of statistical physics and is deeply related to the influence of the disorder on the phase transition in such systems (see e.g. $[48,2]$ and references therein).

\subsection{Quenched central limit theorem.}

The second result concerns the influence of a typical realization of the disorder $\left(\omega_{j}\right)_{j \geq 1}$ on the fluctuations of the empirical measure (4) around its McKean-Vlasov limit (12). The question is whether or not self-averaging also holds at the level of the 
fluctuations, and if not, if it is possible to quantify the dependance in the disorder of the system (3) at the level of fluctuations.

\subsubsection{Non-self-averaging phenomenon in the Kuramoto model}

The motivation comes from the Kuramoto model (5). To fix ideas, consider the case where the frequencies $\omega_{i}$ in (5) are sampled with $\mu=\frac{1}{2}\left(\delta_{-1}+\delta_{1}\right)$ : this is simply a random decomposition of $\left(\theta_{1}, \ldots, \theta_{N}\right)$ between two subpopulations, one naturally rotating clockwise $\left(\omega_{i}=+1\right)$ and the second rotating anti-clockwise $\left(\omega_{i}=-1\right)$. One can imagine that fluctuations in the finite sample $\left(\omega_{1}, \ldots, \omega_{N}\right) \in\{ \pm 1\}^{N}$ may lead, for example, to a majority of +1 with respect to -1 , so that the rotators with positive frequency induce a global rotation of the whole system in the direction of the majority. Direction and speed of rotation depend on this initial configuration of the disorder (see Fig. 1 and 2). This phenomenon, noticed numerically in [5], can be computed through the order parameters $\left(r_{N, t}, \psi_{N, t}\right)$ :

$$
r_{N, t} e^{i \psi_{N, t}}:=\frac{1}{N} \sum_{j=1}^{N} e^{i \theta_{j, t}}=\int_{\mathbf{S} \times \mathbf{R}} e^{i \theta} v_{N, t}(\mathrm{~d} \theta, \mathrm{d} \omega), \quad N \geq 1, t \geq 0,
$$

Here $r_{N, t} \in[-1,1]$ gives a notion of synchronization of the system (5) (e.g. $r_{N, t}=$ 1 if the oscillators $\theta_{j, t}$ are all equal) and $\psi_{N, t}$ captures the position of the center of synchronization (see Figure 1). One can see on Figure 2a that $t \mapsto \psi_{N, t}$ has an approximately linear behavior whose slope depends on the sample of the disorder. Note that this disorder-induced phenomenon does not happen at the level of the nonlinear Fokker-Planck equation (6), but only at the level of fluctuations (the speed of rotation in Figure $2 \mathrm{a}$ is of order $N^{-1 / 2}$ which vanishes as $N \rightarrow \infty$ ). Consequently, in order to understand this phenomenon, one needs to make sense to a quenched central limit theorem for the empirical measure $v_{N}$.

\subsubsection{Weak quenched convergence of the fluctuation process}

We consider, for a fixed realization of the disorder $(\omega)=\left(\omega_{i}\right)_{i \geq 1}$ the fluctuation process $\eta_{N}=\eta_{N}^{(\omega)}$ given by

$$
\eta_{N, t}^{(\omega)}:=\sqrt{N}\left(v_{N, t}^{(\omega)}-v_{t}\right), t \leq T, N \geq 1 .
$$

For fixed $t \leq T,(\omega)$ and $N \geq 1, \eta_{N, t}^{(\omega)}$ is a random element of $\mathscr{S}^{\prime}$, the Schwartz space of tempered distributions on $\mathbf{R}^{m} \times \mathbf{R}^{n}$.

Remark 4. One observation about (16) is that the convergence of $\eta_{N}^{(\omega)}$ as $N \rightarrow \infty$ cannot hold for a fixed realization of $(\omega)$ : consider the particular case of (3) when $m=1, c(\theta, \omega)=\omega, \Gamma=0$ 

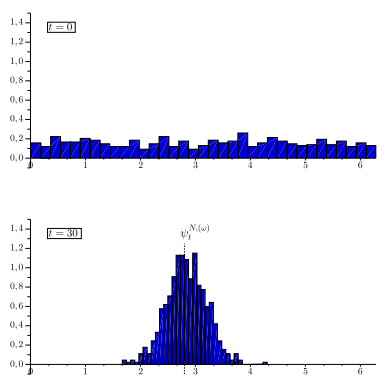
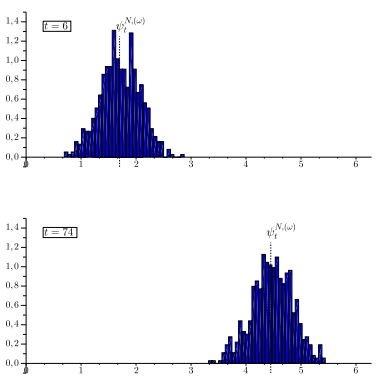

Fig. 1: Evolution of the marginal on $\mathbf{S}$ of $v_{N}$ in the Kuramoto model $(N=600, \mu=$ $\left.\frac{1}{2}\left(\delta_{-1}+\delta_{1}\right), K=6\right)$. The rotators are initially independent and uniformly distributed on $\mathbf{S}$ and independent of the disorder. First the dynamics leads to synchronization $(t=6)$ to a profile close to a nontrivial stationary solution of (6). Second, the center $\psi_{N, t}$ of this density moves to the right with an approximately constant speed; this speed of rotation turns out to be sample-dependent (see Fig. 2a).

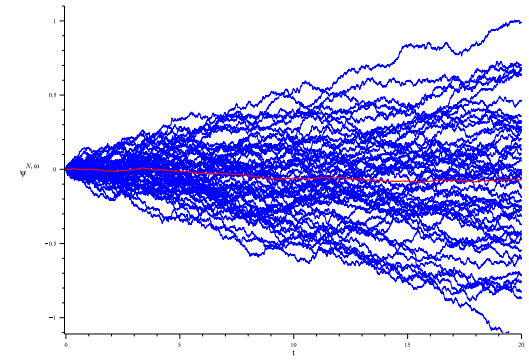

(a) Trajectories of the center of synchronization $\psi_{N}$ for different realizations of the disorder $\left(\omega_{1}, \ldots, \omega_{N}\right)\left(\mu=\frac{1}{2}\left(\delta_{-0.5}+\delta_{0.5}\right)\right.$, $K=4, N=400)$.

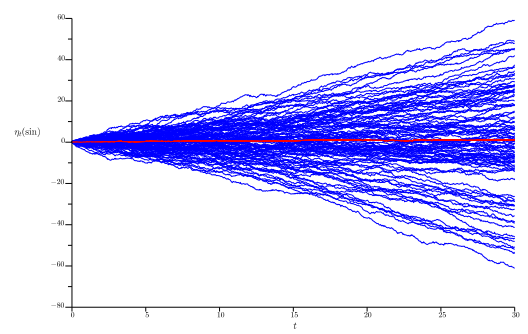

(b) Trajectories of the process $\eta_{t}(\sin )$, for different realizations of the mean-value $C(\omega)$ (see (22)).

Fig. 2: In Fig. 2a, direction and speed of $\psi_{N}$ depend on the initial configuration of the frequencies. These simulations are compatible with speeds of order $N^{-1 / 2}$. In Fig. $2 b$, trajectories of the limiting fluctuation process $\eta(\sin )$ are almost linear and compatible with Fig. 2a. The red trajectories in both figures correspond to the averaged model where there is no disorder-dependent rotation.

$$
\theta_{i, t}=\omega_{i} t+B_{i, t}, t \leq T, i=1, \ldots, N
$$

that is simply Brownian motions with random drifts. Studying the fluctuations of the empirical measure associated to (17) requires to look at functionals of the type $\sqrt{N}\left(\frac{1}{N} \sum_{i=1}^{N} \varphi\left(\omega_{i}\right)-\int \varphi(\omega) \mu(\mathrm{d} \omega)\right)$, for regular functions $\varphi$. But almost-surely in $\left(\omega_{i}\right)_{i \geq 1}$, the latter quantity does not converge (it only converges in law with respect to $\left.\left(\omega_{i}\right)_{i \geq 1}\right)$. 
In order to make sense of any possible limit for $\eta_{N}^{(\omega)}$, one needs to find a weak formulation of a notion of quenched convergence. To do so, fix $(\omega)$ and denote as $\mathscr{H}_{N}(\omega)$ the law of the process $\eta_{N}^{(\omega)} ; \mathscr{H}_{N}(\omega)$ belongs to $\mathscr{M}_{1}\left(\mathscr{C}\left([0, T], \mathscr{S}^{\prime}\right)\right)$, the set of probability measures on continuous paths with values in $\mathscr{S}^{\prime}$. As noted in Remark $4, \mathscr{H}_{N}(\omega)$ is not likely to converge weakly as $N \rightarrow \infty$ for fixed $(\omega)$. Instead, consider the random variable

$$
(\omega) \in\left(\mathbf{R}^{n}\right)^{\mathbf{N}} \mapsto \mathscr{H}_{N}(\omega) \in \mathscr{M}_{1}\left(\mathscr{C}\left([0, T], \mathscr{S}^{\prime}\right)\right) .
$$

The purpose of Theorem 2 below is precisely to state that this random variable (with values in the big set $\left.\mathscr{M}_{1}\left(\mathscr{C}\left([0, T], \mathscr{S}^{\prime}\right)\right)\right)$ converges in law to a random variable $\omega \mapsto \mathscr{H}(\omega) \in \mathscr{M}_{1}\left(\mathscr{C}\left([0, T], \mathscr{S}^{\prime}\right)\right)$. The second point of Theorem 2 is to identify $\mathscr{H}(\omega)$ as the law of the solution to a linear stochastic partial differential equation.

Remark 5. It is important to note that this weak notion of quenched convergence differs from an averaged convergence: it is also possible under the same hypotheses to state an averaged central limit theorem which gives a different limit from the one found in (22) (see [39]). The quenched convergence still keeps track of the dependence in the disorder of the particle system as $N \rightarrow \infty$ (see Theorem 2), whereas the averaged limit does not.

\subsubsection{Assumptions}

In addition to the assumptions of Section 2.1.1, we suppose that

1. The functions $(\theta, \omega) \mapsto c(\theta, \omega)$ and $(\theta, \omega, \tilde{\theta}, \tilde{\omega}) \mapsto \Gamma(\theta, \omega, \tilde{\theta}, \tilde{\omega})$ are infinitely differentiable. The derivatives of $\Gamma$ are uniformly bounded and the derivatives of $c$ satisfy the same polynomial bound as (10).

2. The following moment conditions are satisfied

$$
\int_{\mathbf{R}^{m}}|\theta|^{2(\kappa+\gamma)} \zeta(\mathrm{d} \theta)<\infty \text { and } \int_{\mathbf{R}^{n}}|\omega|^{2(l+\gamma)} \mu(\mathrm{d} \omega)<\infty
$$

where the constants $\kappa$ and $\imath$ are given by (10), for a sufficiently large constant $\gamma$ (depending explicitly on $m, n, \kappa, \imath$ ).

\subsubsection{Quenched central limit theorem}

Before stating the result, let us give some definitions: for all $0 \leq s \leq T$, let $\mathscr{L}_{s}$ be the second order differential operator defined by

$$
\begin{aligned}
\mathscr{L}_{s} f(\theta, \omega): & =\frac{1}{2} \Delta_{\theta} f(\theta, \omega)+\nabla_{\theta} f(\theta, \omega) \cdot\left(\left[\Gamma, v_{s}\right](\theta, \omega)+c(\theta, \omega)\right) \\
& +\int \nabla_{\theta} f(\tilde{\theta}, \tilde{\omega}) \cdot \Gamma(\tilde{\theta}, \tilde{\omega}, \theta, \omega) v_{s}(\mathrm{~d} \tilde{\theta}, \mathrm{d} \tilde{\omega})
\end{aligned}
$$


Let $W$ the Gaussian process with covariance (for every $s, t \in[0, T]$ ):

$$
\mathbf{E}\left(W_{t}\left(f_{1}\right) W_{s}\left(f_{2}\right)\right)=\int_{0}^{s \wedge t} \int \nabla_{\theta} f_{1}(\theta, \omega) \cdot \nabla_{\theta} f_{2}(\theta, \omega) v_{u}(\mathrm{~d} \theta, \mathrm{d} \omega) \mathrm{d} u
$$

For all $f_{1}, f_{2}$ bounded and continuous on $\mathbf{R}^{m} \times \mathbf{R}^{n}$, let

$$
\begin{aligned}
\Gamma_{1}\left(f_{1}, f_{2}\right) & :=\int_{\mathbf{R}^{n}} \operatorname{Cov} \zeta\left(f_{1}(\cdot, \omega), f_{2}(\cdot, \omega)\right) \mu(\mathrm{d} \omega), \\
& =\int_{\mathbf{R}^{n}}\left\{\int_{\mathbf{R}^{m}}\left(f_{1}-\int_{\mathbf{R}^{m}} f_{1}(\cdot, \omega) \mathrm{d} \zeta\right)\left(f_{2}-\int_{\mathbf{R}^{m}} f_{2}(\cdot, \omega) \mathrm{d} \zeta\right) \mathrm{d} \zeta\right\} \mu(\mathrm{d} \omega),
\end{aligned}
$$

and

$$
\begin{aligned}
\Gamma_{2}\left(f_{1}, f_{2}\right) & =\operatorname{Cov}_{\mu}\left(\int_{\mathbf{R}^{m}} f_{1} \mathrm{~d} \zeta, \int_{\mathbf{R}^{m}} f_{2} \mathrm{~d} \zeta\right) \\
& =\int_{\mathbf{R}^{n}}\left(\int_{\mathbf{R}^{m}} f_{1} \mathrm{~d} \zeta-\int_{\mathbf{R}^{m} \times \mathbf{R}^{n}} f_{1} \mathrm{~d} \zeta \mathrm{d} \mu\right)\left(\int_{\mathbf{R}^{m}} f_{2} \mathrm{~d} \zeta-\int_{\mathbf{R}^{m} \times \mathbf{R}^{n}} f_{2} \mathrm{~d} \zeta \mathrm{d} \mu\right) \mathrm{d} \mu
\end{aligned}
$$

The main theorem is the following:

Theorem 2 ([39]). Under the assumptions of Sections 2.1.1 and 2.2.3, the sequence $(\omega) \mapsto \mathscr{H}_{N}(\omega)$ converges in law to the random variable $\omega \mapsto \mathscr{H}(\omega)$, where $\mathscr{H}(\omega)$ is the law of the solution to the Ornstein-Uhlenbeck process $\eta^{\omega}$ solution in $\mathscr{S}^{\prime}$ of the following stochastic partial differential equation:

$$
\eta_{t}^{\omega}=X+C(\omega)+\int_{0}^{t} \mathscr{L}_{s}^{*} \eta_{s}^{\omega} \mathrm{d} s+W_{t}
$$

where, $\mathscr{L}_{s}^{*}$ is the formal adjoint operator of $\mathscr{L}_{s}, X$ is a centered Gaussian process with covariance $\Gamma_{1}$ and where for fixed $\omega, C(\omega)$ is the nontrivial deterministic mean value of the initial condition. As a random variable in $\omega, \omega \mapsto C(\omega)$ is a Gaussian process with covariance $\Gamma_{2}$. Moreover, $W$ is independent on the initial value $(X, C)$.

In (22), the linear operator $\mathscr{L}_{s}^{*}$ is deterministic ; the only dependence in $\omega$ lies in the initial condition $\eta_{0}^{\omega}=X+C(\omega)$, through its non trivial mean-value $C(\omega)$. We give here some intuition about the fact that $C(\cdot)$ precisely captures the fluctuations of the disorder in the microscopic model and show how one can understand from (22) the non-self-averaging behavior of the Kuramoto model described in Section 2.2.1. For $N \geq 1$ and $f: \mathbf{R}^{m} \times \mathbf{R}^{n} \rightarrow \mathbf{R}$, the initial fluctuation applied to $f$ is given by 


$$
\begin{aligned}
\eta_{N, 0}(f) & =\frac{1}{\sqrt{N}} \sum_{j=1}^{N}\left(f\left(\theta_{j, 0}, \omega_{j}\right)-\int_{\mathbf{R}^{m} \times \mathbf{R}^{n}} f(\theta, \omega) \zeta(\mathrm{d} \theta) \mu(\mathrm{d} \omega)\right) \\
& =\frac{1}{\sqrt{N}} \sum_{j=1}^{N}\left(f\left(\theta_{j, 0}, \omega_{j}\right)-\int_{\mathbf{R}^{m}} f\left(\theta, \omega_{j}\right) \zeta(\mathrm{d} \theta)\right) \\
& +\frac{1}{\sqrt{N}} \sum_{j=1}^{N}\left(\int_{\mathbf{R}^{m}} f\left(\theta, \omega_{j}\right) \zeta(\mathrm{d} \theta)-\int_{\mathbf{R}^{m} \times \mathbf{R}^{n}} f(\theta, \omega) \zeta(\mathrm{d} \theta) \mu(\mathrm{d} \omega)\right) \\
& :=X_{N}(f)+C_{N}(f)
\end{aligned}
$$

The process $X_{N}$ captures the initial fluctuations of the rotators whereas $C_{N}$ captures the fluctuations of the disorder. It is easy to see that $C_{N}$ converges in law (w.r.t. the disorder) to the process $C$ with covariance (21). In the framework of the Kuramoto model with binary disorder (recall Section 2.2.1), computations show ([40]) that the relevant quantity for the dynamics of (22) is the restriction $C_{+}$of the process $C$ to the component on +1 :

$$
C_{+, \psi}:=C_{\psi \mathbf{1}_{\omega=+1}}, \quad \psi: \mathbf{S} \rightarrow \mathbf{R} .
$$

$C_{+}$is the limit in law of the microscopic process $C_{N,+}$ defined by

$$
C_{N,+}(\psi):=\left(\int_{\mathbf{S}} \psi(\cdot) \mathrm{d} \zeta\right) \frac{1}{\sqrt{N}} \sum_{i=1}^{N}\left(\mathbf{1}_{\left(\omega_{i}=+1\right)}-\frac{1}{2}\right):=\left(\int_{\mathbf{S}} \psi(\cdot) \mathrm{d} \zeta\right) \frac{\alpha_{N}}{\sqrt{N}} .
$$

Here, $\alpha_{N}$ is exactly the (centered) number of frequencies among $\left(\omega_{1}, \ldots, \omega_{N}\right)$ that are positive, so that $C_{N,+}$ captures the lack of symmetry of the disorder: $\alpha_{N}>0$ (resp. $\alpha_{N}<0$ ) represents the case of an asymmetry in favor of positive (resp. negative) frequencies. Hence, Theorem 2 provides a way to study, through the process $C$, the influence of the asymmetry of the disorder on (3). The main lines of proof of Theorem 2 are given in Section 3.2.

\subsection{Long-time behavior of the fluctuation process in the Kuramoto model}

We restrict in this paragraph to the Kuramoto case (5) with binary disorder: $\mu=$ $\frac{1}{2}\left(\delta_{-\omega_{0}}+\delta_{\omega_{0}}\right)$ for some $\omega_{0}>0$. One can see numerically (see Figure $2 \mathrm{~b}$ ) that the initial asymmetry $C(\cdot)$ propagates from $t=0$ to positive times and provide analogous trajectories to the ones observed in the microscopic model (Figure 2a). One can make this observation rigorous, at least when $\omega_{0}$ is small:

Theorem 3 ([40]). In the Kuramoto model (5) in the small-disorder regime, the solutions of (22) are asymptotically linear and disorder dependent. More precisely, for all $K>1$, there exist an Hilbert space of distributions $\mathbf{H}$ and $\omega^{*}>0$ such that for all $0<\omega_{0}<\omega^{*}$, for fixed initial condition $\eta_{0}^{\omega}=X+C(\omega)$, there exists $V(\omega) \in \mathbf{H}$ 
such that

$$
\frac{\eta_{t}^{\omega}}{t} \stackrel{\text { in law }}{\longrightarrow}=V(\omega), \quad \text { as } t \rightarrow+\infty .
$$

Moreover, $\omega \mapsto V(\omega)$ is a Gaussian process in $\mathbf{H}$ with explicit (in terms of $C(\omega)$ ) covariance.

The proof of Theorem 3 relies on a spectral analysis (based on perturbation argument from the case without disorder, [8]) of the linear operator $\mathscr{L}_{s}^{*}$ governing (22). Following the intuition of the finite-dimensional Jordan block $\left(\begin{array}{l}x \\ y\end{array}\right)^{\prime}=\left(\begin{array}{ll}0 & 1 \\ 0 & 0\end{array}\right) \cdot\left(\begin{array}{l}x \\ y\end{array}\right)$ (whose solutions are obviously linear), one of the key ingredients to the proof of Theorem 3 is to prove a similar property for the unbounded operator $\mathscr{L}_{s}^{*}$ : there exist $u$ and $v$ such that $\mathscr{L}_{s}^{*} u=0$ and $\mathscr{L}_{s}^{*} v=u$. We refer to [40] for more details.

\subsection{Conclusion and perspectives}

Disordered mean-field such as (3) (and especially the Kuramoto model) are not selfaveraging at the level of fluctuations: the dynamics of the quenched fluctuations of (4) is disorder-dependent. However, in order to derive rigorously the exact speed of rotation of synchronized solutions in the Kuramoto model described in Figure 2, it would be necessary to study (5) on larger time scales, as in [9]. This is currently under investigation.

Another difficulty is that, although both law of large numbers and central limit theorem are valid in a rather general setting, investigating the long-time behavior of the limiting objects $v$ and $\eta$ is often very difficult. In that sense, one of the reasons for the popularity of the Kuramoto model is that the stationary solutions of the nonlinear Fokker-Planck equation (6) are explicitly computable ([50]). A key point in the analysis of the stability of such solutions $([8,9,29])$ is that the Kuramoto model without disorder is reversible ([8]), whereas reversibility is lost for many other neuronal models (e.g. FitzHugh-Nagumo [7]). The results presented here should be applicable to other models of disordered diffusions, provided sufficient information is known about characterization and linear stability of stationary states (see for example [38]).

There is currently a growing interest in generalizations of (3) where the interactions depend on the topology of non-mean-field networks or on the distance between particles. The motivation comes from the biological observation that neurons do not interact in a mean-field way (see [55] and references therein). As far as weighted interactions are considered, models of moderately interacting diffusions $([46,35])$ have been one of the first attempts to go beyond pure mean-field models. Several papers in physics [47, 31, 43] have also considered models of oscillators within $P$-nearest neighbors or with power-law interaction. Those models are known to exhibit anomalous dynamical properties (chimera states). The mean-field limit in this framework has been rigorously shown in [41], showing in particular anomalous speed of convergence to the nonlinear Fokker-Planck equation. The exact central limit theorem in this case is currently under investigation. 
Generalizing the result of Dai Pra and den Hollander concerning possible quenched large deviations for (3) is of course a natural perspective for this work and is the object of an ongoing work.

\section{Sketchs of proofs}

\subsection{Law of large numbers}

We give in this paragraph the main lines of the proof of Theorem 1. The difficulty here is that we allow the coefficient $c$ in (3) to have polynomial growth (recall Section 2.1.1); in particular, one needs to have a priori controls on the moments of any accumulation point of (4). The well-posedness of the McKean-Vlasov equation (12) under the hypotheses of Section 2.1.1 can be seen as a consequence of [41]. The existence of a least one solution $v$ (satisfying the required moment conditions) to (12) is established in [41] via a fixed-point procedure on the nonlinear process associated to (12) using arguments from Sznitman [53] and the uniqueness comes from the fact that for any such solution $v$, the empirical measure $v_{N}$ necessarily converges to $v$ as $N \rightarrow \infty$, in terms of an adequate Wasserstein metric. Note that we cannot directly use this last result for the quenched convergence of $v_{N}$, since the convergence in [41] is averaged w.r.t. the disorder.

Remark 6. The framework of [41] concerns the more general case of diffusions within weighted spatial interactions ( $P$-nearest neighbor with parameter $R \in] 0,1]$ and power law with exponent $\alpha \geq 0$, see [41], Section 1.2.2 for detailed definitions of these models). It is easy to see that one retrieves the full mean-field setting for $R=1$ or $\alpha=0$.

As far as the quenched convergence of the empirical measure (4) is concerned, one can proceed as follows. The result for the initial condition is clear: since $\left(\theta_{i, 0}, \omega_{i}\right)_{1 \leq i \leq N}$ are i.i.d. random variables with law $\zeta \otimes \mu$, the initial empirical measure $v_{N, 0}$ converges almost surely in $(\omega)$ to $v_{0}(\mathrm{~d} \theta, \mathrm{d} \omega)=\zeta(\mathrm{d} \theta) \mu(\mathrm{d} \omega)$, as $N \rightarrow \infty$.

An application of Ito's formula to (3) (for any regular function $(\theta, \omega) \mapsto f(\theta, \omega)$ ) leads to the following semi-martingale representation for $v_{N}$ :

$$
\begin{aligned}
\left\langle v_{N, t}, f\right\rangle & =\left\langle v_{N, 0}, f\right\rangle+\int_{0}^{t}\left\langle v_{N, s}, \frac{1}{2} \Delta_{\theta} f+\nabla_{\theta} f \cdot c\right\rangle \mathrm{d} s \\
& +\int_{0}^{t}\left\langle v_{N, s}, \nabla_{\theta} f \cdot \int \Gamma(\cdot, \cdot, \tilde{\theta}, \tilde{\omega}) v_{N, s}(\mathrm{~d} \tilde{\theta}, \mathrm{d} \tilde{\omega})\right\rangle \mathrm{d} s+M_{N, t}(f)
\end{aligned}
$$

where $M_{N, t}(f):=\frac{1}{N} \sum_{j=1}^{N} \int_{0}^{t} \nabla_{\theta} f\left(\theta_{j, s}, \omega_{j}\right) \cdot \mathrm{d} B_{j, s}$ is a martingale. Using usual tightness criteria ([45, 49]) based on Aldous criterion ([10]) for real valued continuous processes, it is easy to derive from (27) the tightness of $\left(v_{N}\right)$ in $\mathscr{C}\left([0, T],\left(\mathscr{M}_{1}\left(\mathbf{R}^{m} \times\right.\right.\right.$ $\left.\left.\mathbf{R}^{n}\right), v\right)$ ), where $v$ is the vague topology (i.e. the coarsest topology that makes the evaluations $v \mapsto\langle v, f\rangle$ continuous for every $f$ continuous with compact support). 
Using the one-sided Lipschitz continuity of $c$, one can prove that, almost surely in $(\omega), \sup _{N \geq 1} \sup _{1 \leq i \leq N} \mathbf{E}\left(\left|\theta_{i, t}\right|^{\kappa}\right)<\infty$. Using this estimate and a localization argument ([39], Lemma 3.4) one obtains that for any accumulation point $\tilde{v}$ of $\left(v_{N}\right)_{N \geq 1}$,

$$
\int_{\mathbf{R}^{m} \times \mathbf{R}^{n}}\left(|\theta|^{\kappa} \vee|\omega|^{l}\right) \tilde{v}(\mathrm{~d} \theta, \mathrm{d} \omega)<+\infty .
$$

The tightness of $\left(v_{N}\right)_{N \geq 1}$ in the weak topology and its convergence to (12) follows from the a priori estimate (28) and the fact that $M_{N, t}(f)$ in (27) vanishes as $N \rightarrow \infty$.

\subsection{Fluctuations}

We now turn to the proof of Theorem 2. The principal tool used here is Hilbertian techniques for measure-values processes developed by Fernandez, Méléard and Jourdain [26, 35] for similar models without disorder.

An application of Ito's formula to (3) leads to a semi-martingale decomposition of the fluctuation process $\eta_{N}^{(\omega)}$ of the following form: for all regular functions $f$, for every sequence $(\omega)$, for all $t \leq T$ :

$$
\left\langle\eta_{N, t}^{(\omega)}, f\right\rangle=\left\langle\eta_{N, 0}^{(\omega)}, f\right\rangle+\int_{0}^{t}\left\langle\eta_{N, s}^{(\omega)}, \mathscr{L}_{N, s}(f)\right\rangle \mathrm{d} s+W_{N, t}^{(\omega)}(f),
$$

where, $\mathscr{L}_{N, s}$ is an unbounded linear operator defined by

$$
\begin{aligned}
\mathscr{L}_{N, s} f(\theta, \omega) & :=\frac{1}{2} \Delta_{\theta} f(\theta, \omega)+\nabla_{\theta} f(\theta, \omega) \cdot\left(\left[\Gamma, v_{N, s}\right](\theta, \omega)+c(\theta, \omega)\right) \\
& +\int \nabla_{\theta} f(\tilde{\theta}, \tilde{\omega}) \cdot \Gamma(\tilde{\theta}, \tilde{\omega}, \theta, \omega) v_{s}(\mathrm{~d} \tilde{\theta}, \mathrm{d} \tilde{\omega}), \theta \in \mathbf{R}^{m}, \omega \in \mathbf{R}^{n} .(30)
\end{aligned}
$$

and $W_{N, t}^{(\omega)}(f)$ is a real continuous martingale with quadratic variation process

$$
\left\langle W_{N}^{(\omega)}(f)\right\rangle_{t}=\int_{0}^{t}\left\langle v_{N, s}^{(\omega)},\left|\nabla_{\theta} f(\theta, \omega)\right|^{2}\right\rangle \mathrm{d} s
$$

Equation (29) is nothing else than a discrete version of the SPDE (22). The natural procedure is then to show that $(\omega) \mapsto \mathscr{H}_{N}(\omega)$ is tight and identify the limit. Since the identification of the limit is standard, we focus on the main difficulty, that is the tightness result.

\subsubsection{The nonlinear process}

The core of the proof consists in introducing the nonlinear process ([35, 42, 53, 52]) associated to the McKean-Vlasov equation (12), that is the diffusion $\bar{\theta}$ whose finite dimensional laws are precisely given by $v_{t}, t \geq 0$. This notion was first introduced by 
Sznitman [53] for systems without disorder. In the context of disordered diffusions, the nonlinear process may be defined as the solution $\left(\bar{\theta}_{t}, \omega\right)_{t \in[0, T]}$ to

$$
\left\{\begin{array}{l}
\bar{\theta}_{t}=\theta_{0}+\int_{0}^{t} c\left(\bar{\theta}_{s}, \omega\right) \mathrm{d} s+\int_{0}^{t}\left[\Gamma, v_{s}\right]\left(\bar{\theta}_{s}, \omega\right) \mathrm{d} s+B_{t} \\
\omega \sim \mu \\
v_{t}=\mathscr{L}\left(\bar{\theta}_{t}, \omega\right), \forall t \in[0, T] .
\end{array}\right.
$$

Note that in (31), $\bar{\theta}$ depends on $v$, which itself is the law of $\bar{\theta}$, so the existence of such a nonlinear process is unclear.

Proposition 1 ([39]). There is pathwise existence and uniqueness in (31).

Proof. The proof follows ideas from Sznitman [53], Th 1.1, p.172. The point is to use a Picard iteration in the space of probabilities on $\mathscr{C}\left([0, T], \mathbf{R}^{m} \times \mathbf{R}^{n}\right)$ endowed with an appropriate Wasserstein metric.

The key point in the proof of the fluctuation theorem (see [26]) is to build a coupling between the particle-system $\left(\theta_{i}\right)_{1<i<N}$ given by (3) and a well-chosen collection of independent nonlinear processes. Namely, for all $i=1, \ldots, N$, consider the nonlinear process $\bar{\theta}_{i}$ defined by (31), with the same initial value as $\theta_{i}$, with the same inhomogeneity $\omega_{i}$, driven by the same Brownian motion $B_{i}$. Then one has

Proposition 2 ( [39]).

$$
\mathbf{E}\left[\sup _{t \leq T}\left|\theta_{i, t}-\bar{\theta}_{i, t}\right|^{2}\right] \leq Z_{N}\left(\omega_{1}, \ldots, \omega_{N}\right)
$$

where the random variable $(\omega) \mapsto Z_{N}(\omega)$ is such that

$$
\lim _{A \rightarrow \infty} \limsup _{N \rightarrow \infty} \mathbb{P}\left(N Z_{N}(\omega)>A\right)=0 .
$$

Proof. By (3) and (31) and using the one-sided Lipschitz continuity of $c$, one has

$$
\begin{aligned}
\mathbf{E}\left[\sup _{s \leq t}\left|\theta_{i, s}-\bar{\theta}_{i, s}\right|^{2}\right] & \leq C\left(\int_{0}^{t} \sup _{1 \leq j \leq N} \mathbf{E}\left[\sup _{u \leq s}\left|\theta_{j, u}-\bar{\theta}_{j, u}\right|^{2}\right] \mathrm{d} s\right. \\
& \left.+\int_{0}^{t} \mathbf{E}\left[\left|\left[\Gamma, \bar{v}_{N, s}-v_{s}\right]\left(\bar{\theta}_{i, s}, \omega_{i}\right)\right|^{2}\right] \mathrm{d} s\right),
\end{aligned}
$$

where $\bar{v}_{N}$ is the empirical measure of the nonlinear processes $\bar{v}_{N, t}:=\frac{1}{N} \sum_{i=1}^{N} \delta_{\left(\bar{\theta}_{i, t}, \omega_{i}\right)}$. Define $T_{i, j, s}:=\Gamma\left(\bar{\theta}_{i, s}, \omega_{i}, \bar{\theta}_{j, s}, \omega_{j}\right)-\int \Gamma\left(\theta_{i, s}, \omega_{i}, \tilde{\theta}, \tilde{\omega}\right) v_{s}(\mathrm{~d} \tilde{\theta}, \mathrm{d} \tilde{\omega})$, so that

$$
\begin{aligned}
\mathbf{E}\left(\left|\left[\Gamma, \bar{v}_{N, s}-v_{s}\right]\left(\bar{\theta}_{i, s}, \omega_{i}\right)\right|^{2}\right) & =\frac{1}{N^{2}} \mathbf{E}\left(\sum_{j=1}^{N}\left|T_{i, j, s}\right|^{2}+\sum_{k \neq l} T_{i, k, s} \cdot T_{i, l, s}\right) \\
& \leq \frac{1}{N^{2}}\left(C N+\mathbf{E}\left(\sum_{\substack{k \neq i, l \neq i \\
k \neq l}} T_{i, k, s} \cdot T_{i, l, s}\right)\right),
\end{aligned}
$$


where we used that $\Gamma$ is bounded. Suppose for a moment that there is no disorder. Taking conditional expectation w.r.t. $\left(\bar{\theta}_{r}, r \neq l\right)$ in the last term and using the exchangeability of the particles, we obtain that this term is zero, leading, by Gronwall's Lemma, to an upper bound in (32) of the form $C / N$ (see [26], Lemma 3.2). Since we work here in a frozen environment (i.e. we do not integrate w.r.t. the disorder and lose the exchangeability of the particles), this additional term becomes nontrivial and not bounded for fixed $(\omega)$. This fact precisely motivates the weak formulation of the quenched convergence introduced in Section 2.2.2. Proof of (33) can be found in [39].

\subsubsection{Weighted Sobolev spaces}

Proposition 2 is the key result in order to show that the random variable $\mathscr{H}_{N}$ defined in Section 2.2.2 is tight. The second tool we use is the introduction of weightedSobolev norms that are specifically adapted to the analysis of (29) (see [26] for a previous similar approach). Namely, for every integer $j, \alpha \geq 0$, we consider the space of all real functions $f$ defined on $\mathbf{R}^{m} \times \mathbf{R}^{n}$ differentiable up to order $j$ such that

$$
\|f\|_{j, \alpha}:=\left(\sum_{\left|k_{1}\right|+\left|k_{2}\right| \leq j} \int_{\mathbf{R}^{m} \times \mathbf{R}^{n}} \frac{\left|D_{\theta}^{k_{1}} D_{\omega}^{k_{2}} f(\theta, \omega)\right|^{2}}{\left(1+|\theta|^{\alpha}\right)^{2}\left(1+|\omega|^{\alpha}\right)^{2}} \mathrm{~d} \theta \mathrm{d} \omega\right)^{1 / 2}<\infty
$$

where, if $k=\left(k_{1}, \ldots, k_{m}\right)$ and $\theta=\left(\theta^{(1)}, \ldots, \theta^{(m)}\right)$, we define $|k|:=\sum_{i=1}^{m} k_{i}$ and $D_{\theta}^{k} h(\theta):=\partial_{\theta^{(1)}}^{k_{1}} \ldots \partial_{\theta^{(m)}}^{k_{m}} h(\theta)$. Let $W_{0}^{j, \alpha}$ be the completion of $\mathscr{C}_{c}^{\infty}\left(\mathbf{R}^{m} \times \mathbf{R}^{n}\right)$ for this norm; $\left(W_{0}^{j, \alpha},\|\cdot\|_{j, \alpha}\right)$ is a Hilbert space and $W_{0}^{-j, \alpha}$ is its dual space. The only thing that differs from the usual Sobolev norm is the weight which is here to control the possibly unbounded coefficient $c$ in (3) and the unbounded disorder. In this framework, using Proposition 2, one can show that under the assumptions of Theorem 2,

Proposition 3. There exist well-chosen Sobolev indices $\left(j_{1}, \alpha_{1}\right)$ and $\left(j_{2}, \alpha_{2}\right)$ such that for fixed $(\omega)$ and $N \geq 1$, for all $T>0$,

1. the imbedding $W_{0}^{-j_{1}, \alpha_{1}} \hookrightarrow W_{0}^{-j_{2}, \alpha_{2}}$ is of Hilbert-Schmidt type,

2. the process $\eta_{N}^{(\omega)}$ is bounded in $W_{0}^{-j_{1}, \alpha_{1}}$ :

$$
\sup _{t \leq T} \mathbf{E}\left[\left\|\eta_{N, t}^{(\omega)}\right\|_{-j_{1}, \alpha_{1}}^{2}\right] \leq A_{N}\left(\omega_{1}, \ldots, \omega_{N}\right),
$$

3. The linear operator $\mathscr{L}_{N, s}$ defined in (30) is continuous from $W_{0}^{j_{2}, \alpha_{2}}$ to $W_{0}^{j_{1}, \alpha_{1}}$, 4. the process $\eta_{N}^{(\omega)}$ is bounded, uniformly in time, in $W_{0}^{-j_{2}, \alpha_{2}}$ :

$$
\mathbf{E}\left[\sup _{t \leq T}\left\|\eta_{N, t}^{(\omega)}\right\|_{-j_{2}, \alpha_{2}}^{2}\right]<B_{N}\left(\omega_{1}, \ldots, \omega_{N}\right) .
$$


Moreover, as random variables in $(\omega), A_{N}$ and $B_{N}$ satisfy

$$
\lim _{A \rightarrow \infty} \limsup _{N \rightarrow \infty} \mathbb{P}\left(A_{N}>A\right)=0 \text {, and } \lim _{A \rightarrow \infty} \limsup _{N \rightarrow \infty} \mathbb{P}\left(B_{N}>A\right)=0 .
$$

\subsubsection{Tightness result}

In the case without disorder (or in the averaged case), the random variables $A_{N}$ and $B_{N}$ would simply be replaced by constants. Using the estimates of Proposition 3 and standard tightness criteria for continuous processes with values in Hilbert spaces (see Joffe and Métivier [33]), proving that the fluctuation process is tight is straightforward ([26]). In the quenched model, $A_{N}$ and $B_{N}$ are not bounded for fixed $(\omega)$. We need to work harder to prove that the random variable $(\omega) \mapsto \mathscr{H}_{N}(\omega)$ is tight in $\mathscr{M}_{1}\left(\mathscr{C}\left([0, T], \mathscr{S}^{\prime}\right)\right)$.

Theorem 4. For all $\varepsilon>0$, there exists a relatively compact subset $K_{\varepsilon}$ in the set $\mathscr{M}_{1}\left(\mathscr{C}\left([0, T], \mathscr{S}^{\prime}\right)\right)$ such that

$$
\limsup _{N \rightarrow \infty} \mathbb{P}\left(\left\{(\omega) ; \mathscr{H}_{N}(\omega) \in K_{\varepsilon}^{c}\right\}\right) \leq \varepsilon
$$

The idea of the proof of Theorem 4 is that, based on Proposition 3, for all $\varepsilon>0$, one can choose $A(\varepsilon)$ sufficiently large so that the quantities in (34) and (35) are smaller than $\varepsilon$ with large probability. $K^{\varepsilon}$ is then constructed as a set of elements in $\mathscr{M}_{1}\left(\mathscr{C}\left([0, T], \mathscr{S}^{\prime}\right)\right)$ satisfying a Joffe and Métivier criterion (hence relatively compact) with parameters (depending on $\varepsilon$ ) chosen in such a way that $\mathscr{H}_{N}(\omega)$ belongs to $K_{\mathcal{E}}$ with high probability. We leave the technical details to [39], Theorem 4.10.

Acknowledgements One of the articles reviewed here ([41]) was written at the Technische Universität of Berlin and was supported by the BMBF, FKZ 01GQ 1001B.

\section{References}

1. J. A. Acebrón, L. L. Bonilla, C. J. Pérez Vicente, F. Ritort, and R. Spigler, The Kuramoto model: A simple paradigm for synchronization phenomena. Rev. Mod. Phys., 77 (2005), 137 185 .

2. M. Aizenman, J. L. Lebowitz, and D. Ruelle, Some rigorous results on the SherringtonKirkpatrick spin glass model, Comm. Math. Phys., 112 (1987), no. 1, 3-20.

3. G. B. Arous and A. Guionnet, Large deviations for Langevin spin glass dynamics, Probab. Theory Rel., 102 (1995), no. 4, 455-509.

4. J. Baladron, D. Fasoli, O. Faugeras, and J. Touboul, Mean-field description and propagation of chaos in networks of Hodgkin-Huxley and FitzHugh-Nagumo neurons, J. Math. Neurosci. (2012), 2:10.

5. N. J. Balmforth and R. Sassi, A shocking display of synchrony, Physica D: Nonlinear Phenomena (2000), 143, 21-55.

6. G. Ben Arous and A. Guionnet, Symmetric Langevin spin glass dynamics, Ann. Probab. (1997), 1367-1422. 
7. N. Berglund and D. Landon, Mixed-mode oscillations and interspike interval statistics in the stochastic Fitzhugh-Nagumo model, Nonlinearity (2012), 252303.

8. L. Bertini, G. Giacomin, and K. Pakdaman, Dynamical aspects of mean field plane rotators and the Kuramoto model, J. Statist. Phys. (2010), 138, 270-290.

9. L. Bertini, G. Giacomin, and C. Poquet, Synchronization and random long time dynamics for mean-field plane rotators, Probab. Theory Rel. (2013), 1-61.

10. P. Billingsley, Convergence of probability measures, Wiley Series in Probability and Statistics: Probability and Statistics. John Wiley \& Sons Inc., New York, second edition, (1999).

11. P. Blanchard, M. Röckner, and F. Russo, Probabilistic representation for solutions of an irregular porous media type equation, Ann. Probab. (2010), 38:1870-1900.

12. F. Bolley, I. Gentil, and A. Guillin, Uniform convergence to equilibrium for granular media, Archive for Rational Mechanics and Analysis (2012), 1-17.

13. F. Bolley, A. Guillin, and C. Villani, Quantitative concentration inequalities for empirical measures on non-compact spaces, Probab. Theory Rel. (2007), 137, 541-593.

14. T. Cabana and J. Touboul, Large deviations, dynamics and phase transitions in large stochastic heterogeneous neural networks (2013), arXiv:1302.6951.

15. M. J. Cáceres, J. A. Carrillo, and B. Perthame, Analysis of nonlinear noisy integrate \& fire neuron models: blow-up and steady states, J. Math. Neurosci. (2011), 1, 33.

16. F. Collet and P. Dai Pra, The role of disorder in the dynamics of critical fluctuations of mean field models, Electron. J. Probab. (2012), 17 no. 26, 40.

17. F. Collet, P. Dai Pra, and E. Sartori, A simple mean field model for social interactions: dynamics, fluctuations, criticality, J. Stat. Phys. (2010), 139, 820-858.

18. P. Dai Pra and F. den Hollander, McKean-Vlasov limit for interacting random processes in random media, J. Statist. Phys. (1996), 184735-772.

19. D. A. Dawson and J. Gärtner, Large deviations from the McKean-Vlasov limit for weakly interacting diffusions, Stochastics (1987), 20,247-308.

20. P. Degond, A. Frouvelle, and J.-G. Liu, Macroscopic limits and phase transition in a system of self-propelled particles, J. Nonlin. Sci. (2012), 1-30.

21. F. Delarue, J. Inglis, S. Rubenthaler, and E. Tanré, Global solvability of a networked integrateand-fire model of McKean-Vlasov type (2012), arXiv:1211.0299.

22. F. Delarue, J. Inglis, S. Rubenthaler, and E. Tanré, Particle systems with a singular mean-field self-excitation, Application to neuronal networks (2014), arXiv:1406.1151.

23. G. B. Ermentrout and D. H. Terman, Mathematical foundations of neuroscience, volume 35 of Interdisciplinary Applied Mathematics, Springer, New York, (2010).

24. M. Fathi, A gradient flow approach to large deviations for diffusion processes (2014), arXiv: 1405.3910 .

25. O. Faugeras and J. MacLaurin, A large deviation principle for networks of rate neurons with correlated synaptic weights (2013), arXiv:1302.1029.

26. B. Fernandez and S. Méléard, A Hilbertian approach for fluctuations on the McKean-Vlasov model, Stochastic Process. Appl. (1997), 71, 33-53.

27. J. Gärtner, On the McKean-Vlasov limit for interacting diffusions, Math. Nachr. (1988), 137 197-248.

28. G. Giacomin, E. Luçon, and C. Poquet, Coherence stability and effect of random natural frequencies in populations of coupled oscillators, J. Dynam. Differential Equations (2014), 26, 333-367.

29. G. Giacomin, K. Pakdaman, and X. Pellegrin, Global attractor and asymptotic dynamics in the Kuramoto model for coupled noisy phase oscillators, Nonlinearity (2012), 25, 1247.

30. D. Godinho and C. Quininao, Propagation of chaos for a sub-critical Keller-Segel model, to appear in Ann. Inst. H. Poincaré Probab. Statist. (2013), arXiv:1306.3831.

31. S. Gupta, M. Potters, and S. Ruffo, One-dimensional lattice of oscillators coupled through power-law interactions: Continuum limit and dynamics of spatial fourier modes. Phys. Rev. E (2012), 85066201.

32. M. Hitsuda and I. Mitoma, Tightness problem and stochastic evolution equation arising from fluctuation phenomena for interacting diffusions, J. Multivariate Anal. (1986), 19, 311-328. 
33. A. Joffe and M. Métivier, Weak convergence of sequences of semimartingales with applications to multitype branching processes, Adv. in Appl. Probab. (1986), 18, 20-65.

34. B. Jourdain and F. Malrieu, Propagation of chaos and Poincaré inequalities for a system of particles interacting through their CDF, Ann. Appl. Probab. (2008), 18, 1706-1736.

35. B. Jourdain and S. Méléard, Propagation of chaos and fluctuations for a moderate model with smooth initial data, Ann. Inst. H. Poincaré Probab. Statist. (1998), 34, 727-766.

36. B. Jourdain and J. Reygner, Capital distribution and portfolio performance in the mean-field atlas model (2013), arXiv:1312.5660.

37. Y. Kuramoto, Self-entrainment of a population of coupled non-linear oscillators, In International Symposium on Mathematical Problems in Theoretical Physics (Kyoto Univ., Kyoto) (1975), 420-422. Lecture Notes in Phys., 39. Springer, Berlin.

38. S. Louca and F. M. Atay, Stationary states in infinite networks of spiking oscillators with noise, SIAM J. Appl. Dyn. Syst. (2013), 12, 415-449.

39. E. Luçon, Quenched limits and fluctuations of the empirical measure for plane rotators in random media, Electr. J. Prob. (2011), 16, 792-829.

40. E. Luçon, Large time asymptotics for the fluctuation SPDE in the Kuramoto synchronization model, J. Funct. Anal. (2014), 266, 6372 - 6417.

41. E. Luçon and W. Stannat, Mean field limit for disordered diffusions with singular interactions, Ann. Appl. Probab. (2014), 24, 1946-1993.

42. F. Malrieu, Convergence to equilibrium for granular media equations and their Euler schemes, Ann. Appl. Probab. (2003), 13, 540-560.

43. M. Maródi, F. d'Ovidio, and T. Vicsek, Synchronization of oscillators with long range interaction: Phase transition and anomalous finite size effects, Phys. Rev. E (2002), 66011109.

44. H. P. McKean, Jr, Propagation of chaos for a class of non-linear parabolic equations, In Stochastic Differential Equations (Lecture Series in Differential Equations, Session 7, Catholic Univ., 1967), (1967) 41-57. Air Force Office Sci. Res., Arlington, Va..

45. S. Méléard and S. Roelly, Sur les convergences étroite ou vague de processus à valeurs mesures, C. R. Acad. Sci. Paris Sér. I Math. (1993), 317, 785-788.

46. K. Oelschläger, A law of large numbers for moderately interacting diffusion processes, Z. Wahrsch. Verw. Gebiete (1985), 69, 279-322.

47. I. Omelchenko, Y. Maistrenko, P. Hövel, and E. Schöll, Loss of coherence in dynamical networks: Spatial chaos and chimera states, Phys. Rev. Lett. (2011), 106234102.

48. L. Pastur and M. Shcherbina, Absence of self-averaging of the order parameter in the Sherrington-Kirkpatrick model, J. of Stat. Phys. (1991), 62, 1-19.

49. S. Roelly-Coppoletta, A criterion of convergence of measure-valued processes: application to measure branching processes, Stochastics (1986), 17, 43-65.

50. H. Sakaguchi, S. Shinomoto, and Y. Kuramoto, Phase transitions and their bifurcation analysis in a large population of active rotators with mean-field coupling, Progr. Theoret. Phys. (1988), 79 600-607.

51. S. H. Strogatz and R. E. Mirollo, Stability of incoherence in a population of coupled oscillators, J. Statist. Phys. (1991), 63 613-635.

52. A.-S. Sznitman, Nonlinear reflecting diffusion process, and the propagation of chaos and fluctuations associated, J. Funct. Anal. (1984), 56 311-336.

53. A.-S. Sznitman, Topics in propagation of chaos, In École d'Été de Probabilités de Saint-Flour XIX - 1989, volume 1464 of Lecture Notes in Math. (1991), 165-251. Springer, Berlin.

54. J. Touboul, Limits and dynamics of stochastic neuronal networks with random heterogeneous delays, J. Stat. Phys. (2012), 149 569-597.

55. G. Wainrib and J. Touboul, Topological and dynamical complexity of random neural networks, Phys. Rev. Lett. (2013), 110118101. 